\title{
Hubble Space Telescope Observations of Comet 9P/Tempel 1 during the Deep Impact Encounter
}

\author{
Paul D. Feldman, Stephan R. McCandliss, Matthew Route \\ Department of Physics and Astronomy, The Johns Hopkins University \\ Charles and 34th Streets, Baltimore, MD 21218-2695 \\ pdf@pha.jhu.edu \\ Harold A. Weaver \\ Space Department, Johns Hopkins University Applied Physics Laboratory, \\ 11100 Johns Hopkins Road, Laurel, MD 20723-6099 \\ Michael F. A'Hearn \\ Department of Astronomy, University of Maryland, \\ College Park MD 20742-2421 \\ Michael J. S. Belton \\ Belton Space Exploration Initiatives, Tucson, AZ 85716 \\ Karen J. Meech \\ University of Hawaii, Institute for Astronomy and \\ NASA Astrobiology Institute, \\ 2680 Woodlawn Drive, Honolulu, HI 96822
}

\begin{abstract}
We report on the Hubble Space Telescope program to observe periodic comet 9P/Tempel 1 in conjunction with NASA's Deep Impact mission. Our objectives were to study the generation and evolution of the coma resulting from the impact and to obtain wide-band images of the visual outburst generated by the impact. Two observing campaigns utilizing a total of 17 HST orbits were carried out: the first occurred on 2005 June 13-14 and fortuitously recorded the appearance of a new, short-lived fan in the sunward direction on June 14. The principal campaign began two days before impact and was followed by contiguous orbits through impact plus several hours and then snapshots one, seven, and twelve
\end{abstract}


days later. All of the observations were made using the Advanced Camera for Surveys (ACS). For imaging, the ACS High Resolution Channel (HRC) provides a spatial resolution of $36 \mathrm{~km}\left(16 \mathrm{~km} \mathrm{pixel}^{-1}\right)$ at the comet at the time of impact. Baseline images of the comet, made prior to impact, photometrically resolved the comet's nucleus. The derived diameter, $6.1 \mathrm{~km}$, is in excellent agreement with the $6.0 \pm 0.2 \mathrm{~km}$ diameter derived from the spacecraft imagers. Following the impact, the HRC images illustrate the temporal and spatial evolution of the ejecta cloud and allow for a determination of its expansion velocity distribution. One day after impact the ejecta cloud had passed out of the field-of-view of the HRC.

Subject headings: comets, 9P/Tempel 1; Deep Impact

\section{INTRODUCTION}

The Deep Impact mission (A'Hearn et al. 2005a,b) successfully placed a $364 \mathrm{~kg}$, about half copper, impactor, onto the surface of comet $9 / \mathrm{P}$ Tempel 1 at a relative velocity of $10.3 \mathrm{~km} \mathrm{~s}^{-1}$ on 2005 July 4 at 05:52:03 UT (as seen from Earth). The event was observed by cameras aboard the mother spacecraft and by a large number of Earth- and space-based telescopes as part of an extensive campaign to study the comet prior to, during, and in the course of several days following the impact (Meech et al. 2005). The Hubble Space Telescope (HST) provided the highest spatial resolution images from Earth $\left(36 \mathrm{~km}, 16 \mathrm{~km} \mathrm{pixel}{ }^{-1}\right)$ using the Advanced Camera for Surveys (ACS) High Resolution Channel (HRC), and also ultraviolet imaging using the Solar Blind Channel (SBC) of ACS to study the evolution of gaseous species, particularly CO, released by the impact. This paper describes the HST campaign and presents the images obtained and the preliminary interpretation of the data in terms of the physical properties of the observed ejecta. We also compare the ejecta produced by the impact with that from a "natural outburst" that was serendipitously observed on 2005 June 14 .

\section{OBSERVATIONS}

The HST campaign consisted of 17 separate "visits", each comprising a single HST orbit allowing $\sim 53$ minutes of target visibility per orbit. Twelve of these orbits were dedicated to visible imaging with the HRC and they are summarized in Table 1. Four of the visits

(program ID 10144) were on 2005 June 13-14, three weeks before impact, and spanned a 
full rotation (40.7 $\mathrm{h}$ ) of the nucleus. The remainder (program ID 10456) were grouped into three periods: a pre-impact group beginning roughly one cometary rotation before impact to establish a baseline for the data to follow; an orbit that included the impact time; and several orbits immediately following the impact and continuing, with single orbits, 7 and 12 days after the impact. The importance of the HST observations was underscored by the coordination between the STScI and JPL to adjust the impact time to allow for HST visibility of the event. As it turned out, impact occured 15 minutes before the comet set below HST's horizon.

All of the visible imaging was done with the HRC using a broad V filter, F606W, to maximize the sensitivity to faint coma structures. This filter is centered at a wavelength of $5907 \AA$ and has a bandpass of $2342 \AA$. ${ }^{1}$ The observing program, with the exception of the impact orbit, consisted of pairs of long (140 or $300 \mathrm{~s}$ ) and short (40 s) exposures interlaced with objective prism images (PR200L) that have so far been difficult to interpret. For the impact orbit, a continuous sequence of 40 second exposures using the HRC $512 \times 512$ pixel mode (half-frame; the full-frame $1024 \times 1024$ pixel mode was used for all other July observations) was used to avoid the possibility of filling the data buffer and requiring a buffer dump at the time of impact. With overhead included, the images were taken at 75 -second intervals. The impact occurred almost exactly in the middle of exposure J9A805EBQ. A total of 12 exposures were taken including and following impact.

Because of the large overhead in switching cameras during a single HST orbit, entire visits were dedicated to the SBC observations. The results of these observations are being reported separately (Feldman et al. 2006).

\section{VISUAL IMAGING}

All of the individual images were processed with pipeline software to produce flat-fielded, geometrically corrected output products that were then rotated to orient celestial north up. An example of the nature of the appearance of the ejecta plume is shown in Fig. 1 in which the right-hand image, a composite of two 140-s exposures, was taken about one hour after impact while the left-hand image, a composite of four 140-s exposures, was taken 54 hours

\footnotetext{
${ }^{1}$ These parameters are from the ACS Instrument Handbook available at http://www.stsci.edu/hst/acs/documents/handbooks/cycle15/c05_maging2.html\#357257. For purposes of photometric calibration, for the F606W filter used with the HRC, Sirianni et al. (2005) define a pivot wavelength of $5888 \AA$ and a width of $665 \AA$, where the latter includes the system throughput as a multiplicative factor.
} 
earlier. The pre-impact image is dominated by light from the nucleus and the inner coma (because the brightness of the quiescent coma falls as the inverse of the distance from the nucleus) while the post-impact image shows clearly the extended fan of ejected material. To enhance the effects of the impact, the remainder of the images presented will be the ratio of a given image to a pre-impact image. The false color scale will then correspond to the relative increase of material in the coma as a result of the impact relative to its quiescent state.

\subsection{Pre-impact Images}

\subsubsection{Radial Profiles and the Photometric Resolution of the Nucleus}

The HRC images of the quiescent comet are dominated by a strong central peak, surrounded by the dust coma, which while not perfectly azimuthally symmetric, nevertheless closely follows a $\rho^{-1}$ brightness distribution as a function of distance from the nucleus. The

width of the bright peak closely matches the camera's point spread function (PSF) (FWHM of 2.25 pixels or $\sim 36 \mathrm{~km}$ projected at the comet for the July observations). Most of the signal is from the nucleus, which is not spatially resolved. For comparison with the images from the Deep Impact spacecraft, we used the last HRC image prior to impact, J9A805EAQ, to evaluate the photometric area of the nucleus. An azimuthally symmetric coma model, convolved with the instrument PSF, was subtracted from the image and the azimuthally averaged radial profile extracted, as has been done for many comets observed by HST (Lamy et al. 2001, 2004). The result is shown in Fig. 2, where the nucleus is found to account for $\sim 90 \%$ of the counts in the central pixel. Using the recent ACS calibration data of Sirianni et al. (2005), the $\mathrm{V}$ magnitude of the nucleus is 17.49. Assuming an average geometric albedo of $4.0 \%$ and a phase law of $0.04 \mathrm{mag} \mathrm{deg}^{-1}$ (Lamy et al. 2004), this magnitude implies an effective diameter of $6.1 \mathrm{~km}$. Direct imaging of the nucleus from Deep Impact gives a diameter of $6.0 \pm 0.2 \mathrm{~km}$ (A'Hearn et al. 2005b), which suggests that our choices for the albedo and phase law are accurate.

\subsubsection{The June 14 Outburst}

Our intention for the June visits was to obtain HRC images evenly spaced over a single rotation period, $40.7 \mathrm{~h}$ (A'Hearn et al. 2005b), to compare with images taken by the spacecraft cameras that would yield comparable spatial resolution at the distance of the comet. It was also an opportunity to test our strategy of taking half-frame images during the impact 
orbit and verify the ephemeris. The four visits are summarized in Table 1. The fourth visit shows a markedly different coma shape from that seen in the preceding visits, and the ratio of these images to one from the third visit, 7 hours earlier, is shown in Fig. 3. The ratio images were created from sums of two 300-second exposures. Fortuitously, the fourth visit was split over two HST orbits so that the two images shown in the figure were taken 67 minutes apart. The expansion of the outburst cone, filling nearly the entire sunward hemisphere, is clearly seen by comparison of the two images, shown as contour plots in Fig. 4. The outer isophote in each figure is well matched by a semi-circle centered at the nucleus, suggesting uniform radial expansion into the sunward hemisphere. The velocity of this isophote, projected onto the plane of the sky, is $145 \mathrm{~m} \mathrm{~s}^{-1}$, which for a hemispherical shell represents a true expansion velocity. As this isophote contains $98.5 \%$ of the additional light produced by the outburst, the derived velocity is a good approximation to the maximum expansion velocity of the outflowing material. For a hemispherical shell, the peak brightness occurs at the inner boundary of the shell, which leads to a minimum velocity of $60 \mathrm{~m} \mathrm{~s}^{-1}$. The similar morphology of the contours in Fig. 4 suggests that the shell is expanding uniformly and that the event that produced the shell was short-lived relative to the time since the outburst.

Extrapolating backwards to the nucleus, assuming constant velocity, the outburst occurred $\sim 4.5$ hours before the mid-point of the left exposure of Fig. 3, or at $\sim 09: 45$ UT. This time appears to be consistent with the same event seen by the imagers on Deep Impact, following a re-analysis of the data presented by A'Hearn et al. (2005b) and is also the same event that was reported by Lara et al. (2006), who observed it at $\sim 21: 00$ UT on June 14 . A duration time of the event, $\sim 10$ minutes, deduced for several natural outbursts from the Deep Impact light curve (Fig. 4 of A'Hearn et al. 2005b), is consistent with the analysis presented above.

\subsection{Impact Orbit}

As noted above, for the impact orbit, a sequence of 40-second exposures using the HRC $512 \times 512$ pixel mode was taken at $75 \mathrm{~s}$ intervals. The impact occurred almost exactly in the middle of exposure J9A805EBQ. A total of 12 exposures were taken including and following impact and these are shown, as ratios to the mean of the last four exposures prior to impact in Fig. 5. The expansion of the ejecta is illustrated by contour plots of four of these ratio images given in Fig. 6. These plots illustrate the initial expansion of the ejecta, characterized by a steady increase in brightness in the vicinity of the nucleus, together with an apparent elongation and displacement towards the southeast. This is in a direction opposite from a position angle between that of the projection of the impactor velocity vector on the sky 
$\left(355^{\circ}\right)$ and that of its downrange component tangent to the comet's surface $\left(294^{\circ}\right)$. These directions, as seen from Earth, are given by Carcich and Elliott (2006) from a recent shape model derived from the in situ imaging. The brightening in the uprange direction may be the later evolution of a highly foreshortened uprange plume seen in the first second after impact

by the Deep Impact Medium Resolution Imager (A'Hearn et al. 2005b). These authors note that such a plume is similar to plumes observed in the laboratory in which the target material is porous (Schultz et al. 2005).

The outermost contour in the SE quadrant of the final image of the sequence (J9A805EMQ) corresponds to an expansion velocity of $390 \mathrm{~m} \mathrm{~s}^{-1}$, assuming that the observed material was generated at impact time. Taking the difference of this image with a pre-impact image, we find that $10 \%$ of the additional light produced by the impact corresponds to material with velocities higher than this, and $1 \%$ corresponds to velocities $\geq 1.5 \mathrm{~km} \mathrm{~s}^{-1}$. This last component is likely due to material produced in the initial ejecta plume (A'Hearn et al. 2005b). Further discussion of these images, based on the light curves derived from aperture photometry, is given below.

\subsection{Post-impact Images and Dust Expansion Velocity}

On all of the HST orbits dedicated to HRC imaging post-impact, pairs of images were taken with exposure times of 40 and 140 seconds each to insure against possible saturation of the image due to a bright ejecta plume near the nucleus. The quiescent level of the comet was established from the June observations. These were interspersed with prism images, so that each orbit can be represented by a pair of images, each of $280 \mathrm{~s}$ exposure time, separated by 24 minutes. In this way, the temporal development of the ejecta morphology may be illustrated, as in Fig. 7, which shows the ratio images from visit 06 beginning about one hour after impact. Most striking in this pair of images is the expansion of the ejecta "fan" in the SW quadrant, in contrast to the images obtained during the 15 minute period following the impact. The fan is symmetric about a position angle of $240^{\circ}$, which is close to the direction of the normal to the surface of the comet at the impact site as seen from Earth (position angle $233^{\circ}$ ) according to the comet shape model of Carcich and Elliott (2006). This fan geometry is characteristic of the larger scale ground-based images that were obtained during the Deep Impact campaign (Schleicher et al. 2006; Sugita et al. 2005).

A similar pair, from an orbit $3.2 \mathrm{~h}$ later (visit 08), is shown in Fig. 8. Here the morphology is almost identical except that the scale of the images is about four times larger. This implies uniform expansion of the ejecta with a distribution of velocities that remains constant with time. As in the case of the June 14 outburst, the maximum expansion velocity, 
associated with the smallest grains, can be determined from the differences in the outermost contour in the two panels derived from Fig. 7, shown in Fig. 9. The contours are well matched by semi-circular isophotes, except for the NW quadrant where the effects of solar radiation pressure are beginning to be felt (Schleicher et al. 2006). The outermost isophote contains $97 \%$ of the light produced by the impact in the SW quadrant. The velocity derived from this isophote is $280 \mathrm{~m} \mathrm{~s}^{-1}$ and this value is consistent with the extrapolation to both the impact time and to the outer contours of the images in Fig. 8. For an expanding hemispherical shell, the inner boundary of the shell, corresponding to the slowest moving particles, is at the position of the brightest contour. This corresponds to a velocity of $\sim 80 \mathrm{~m} \mathrm{~s}^{-1}$. In the images taken immediately following impact (Fig. 6), the contours in the SW quadrant also appear to be expanding with a velocity of $\sim 300 \mathrm{~m} \mathrm{~s}^{-1}$, suggesting that any acceleration of the excavated material must take place within $100 \mathrm{~km}$ of the nucleus.

If we assume that the time during which material is ejected is short compared to some later time after the event, then the radial brightness profile will reflect the velocity distribution of the ejected material. In Fig. 10 we show radial profiles of both the pre- and post-impact images used in the ratio images of Fig. 7. These profiles are generated by integrating the detector counts along annuli between position angles $\pm 60^{\circ}$ from the symmetry axis. The difference between these profiles, multiplied by the radius (in pixel units), is shown in blue. The dashed curve indicates what fraction of the flux lies outside a given radial distance.

The blue curves in Fig. 10 are converted to velocity by dividing by the time difference between the exposure mid-point and the impact time. They are then normalized to unity with respect to integration over velocity, and are shown in Fig. 11. The two distribution functions, based on images with exposure mid-points 68.2 and 92.2 minutes, respectively, after the impact, are in good agreement, the slight differences likely due to the finite time for particle ejection (estimated to be $4-5$ minutes), and the fact that the integration over velocity does not fully extend to infinity. With these distribution functions nearly identical results are obtained for the mean expansion speed, $115 \mathrm{~m} \mathrm{~s}^{-1}$, and rms velocity, $145 \mathrm{~m} \mathrm{~s}^{-1}$. The most probable velocity is $70-80 \mathrm{~m} \mathrm{~s}^{-1}$, as found from the contour plots. The orbit $3.2 \mathrm{~h}$ later (Fig. 8) gives a qualitatively similar result but is affected by the finite size of the detector.

Our results may be compared with a number of other estimates of dust expansion velocity. From visible imaging, Schleicher et al. (2006) report a maximum velocity of $230 \mathrm{~m} \mathrm{~s}^{-1}$, while Küppers et al. (2005), from images taken with the OSIRIS camera on Rosetta, report a typical velocity of $110 \mathrm{~m} \mathrm{~s}^{-1}$ with a maximum of $300 \mathrm{~m} \mathrm{~s}^{-1}$. Sugita et al. (2005), from 8-13 $\mu \mathrm{m}$ images, derive a mean expansion velocity of $125 \pm 10 \mathrm{~m} \mathrm{~s}^{-1}$, which is consistent with 
the larger particles seen in the infrared. We also note that our derived velocity is about twice as large as that seen in the June 14 outburst, an event presumably driven by the sublimation of volatile ices, so that the energy for the plume expansion must have come mainly from the impact.

The angular distribution of the ejecta material about the normal to the surface of the comet at the impact site provides information about the nature of the cratering process. A significant change in morphology is seen between the images taken during the first 14 minutes

after impact (Fig. 5) and those beginning an hour after impact (Fig. 7). An example of the azimuthal distribution of the ejecta in this latter case is shown in Fig. 12.

By the time of the next visit with HRC exposures, $\sim 19$ h after impact, the ejecta plume had expanded beyond the $29^{\prime \prime} \times 26^{\prime \prime}$ field-of-view of the camera. The brightness surrounding the nucleus remains higher than in the pre-impact images because the line-of-sight passes through the ejecta cone in front of and behind the plane of the sky.

\subsection{Light Curves}

Aperture photometry was generated from the images by integrating the detector count rate over a range of circular apertures with radii from 2.5 pixels $(40 \mathrm{~km})$ to 250 pixels (4050 km) centered on the comet's nucleus. The data from the impact orbit (visit 05) are shown in the left-hand panel of Fig. 13, in which an average of pre-impact points has been subtracted from the count rate integrated over each aperture. The impact flash may be responsible for the small signal rise in the image containing the impact event. This is followed by a sharp increase in all of the apertures which then continue to increase monotonically with time. The increase in the three smallest apertures is a factor of 8-10 over the pre-impact count rate in a 13.7 minute period. The upward curvature seen in the larger apertures may be the result of the fragmentation of the ejected grains into smaller particles. The near-linear rise in the $40 \mathrm{~km}$ radius aperture is likely due to decreasing optical depth in the central region of the aperture so that the effective scattering surface is increasing as the material approaches optical depth unity. There is no evidence in these data for a dip in brightness increase at $\sim 200 \mathrm{~s}$, as reported by Keller et al. (2005) from OSIRIS images, but this discrepancy may be the result of the different viewing geometry from Earth and from the Rosetta spacecraft.

The right-hand panel of the figure shows that activity had ceased by 60 minutes after impact and that the ejecta had effectively moved out of the fields-of-view of the three smallest apertures. Only the $400 \mathrm{~km}$ radius aperture shows an increase from the last data point in the left-hand panel, implying that the velocity of the slowest moving ejecta particles was 
$<135 \mathrm{~m} \mathrm{~s}^{-1}$. From the $160 \mathrm{~km}$ aperture, we also find this velocity must be $>55 \mathrm{~m} \mathrm{~s}^{-1}$, consistent with the value found from the contour analysis above. The 49 minute data gap makes it difficult to further constrain these values.

A lightcurve through 20 hours after impact, giving the observed count rates in the larger apertures to facilitate comparison with ground-based data, is shown in Fig. 14. By the time of the following $H S T$ visit, 7.5 days after impact, the comet had returned to its pre-impact level of activity and the photometry showed a slow decrease of count rate with time in all of the apertures. As in Fig. 13, the shape of the light curve is a function of aperture. Comparison with the $10.5 \mu \mathrm{m}$ light curves of Sugita et al. (2005) also suggests that the light curves are a function of wavelength, as the infrared is mostly sensitive to larger, slower moving grains. In our case, we note that the total flux, as measured in the 200 and 250 pixel radius apertures appears to remain constant from about 1 to 5 hours after impact. We use the count rates in these apertures and the photometric calibration of Sirianni et al. (2005) to estimate the total $V$-band flux from the ejecta plume as $1.11 \times 10^{-14} \mathrm{ergs} \mathrm{cm}^{-2} \mathrm{~s}^{-1} \AA^{-1}$ at $5888 \AA$, which corresponds to a $V$ magnitude of 13.7 .

\subsection{Dust Content}

From the derived total flux we can estimate the effective surface area of the ejected material, which together with an assumption about the size distribution of the grains can provide a constraint on the total mass of the solid ejecta. Using the same geometric albedo (0.04) and phase function (0.04 mag $\left.\mathrm{deg}^{-1}\right)$ that was used to derive the photometric size of the nucleus, the observed flux corresponds to an area of $960 \mathrm{~km}^{2}$. If instead we use the assumptions of Küppers et al. (2005), a scattering albedo of 0.10 (corresponding to a geometric albedo of 0.025, Hanner et al. 1981), and no phase function, we find an area of $340 \mathrm{~km}^{2}$, in excellent agreement with the value of $(330 \pm 30) \mathrm{km}^{2}$ quoted by Küppers et al. The semi-circular area of the 200 pixel radius aperture is $1.65 \times 10^{7} \mathrm{~km}^{2}$, so the filling factor is $\leq 10^{-4}$ and optical depth effects can be neglected. We can also compare this result with the total flux observed following the natural outburst of June 14 (Fig. 3) which we find to be smaller by a factor of 7.1. Correcting for the heliocentric and geocentric distances on the two dates, the ratio of ejected material is 8.6. We can scale the impactor mass by this factor, and find that a meteoroid of $\sim 40 \mathrm{~kg}$ mass, at the velocity of Deep Impact, would be needed to produce such an outburst, quite unlikely in view of the frequent nature and apparent periodicity of the outbursts (A'Hearn et al. 2005b), in agreement with the conclusion reached by Küppers et al.

While it is possible to use the derived area to calculate the total ejecta volume (and then 
mass) by assuming a power law distribution of particle sizes, visible imaging samples only a small portion of the total mass distribution so that small uncertainties in the assumed size distribution can lead to large differences in the final results. This accounts for the discrepancy between the mass derived by Küppers et al. and smaller values derived from infrared imaging and spectroscopy (Sugita et al. 2005; Harker et al. 2005). It is also possible to use the rms expansion velocity derived above to constrain the total mass using energy arguments provided that we can determine what fraction of the kinetic energy of the impactor is transferred to the solid ejecta during the excavation of the crater. However, we note the caveat that the velocity distribution of Fig. 11 is only an approximate velocity distribution because it is weighted by the velocity dependence of the mass (and consequently, the area) of the scattering particles. Thus, detailed modeling, drawing upon additional imaging data in other spectral bands, is needed to properly address this issue.

\section{CONCLUSION}

We have presented an overview of the visible imaging obtained with the Advanced Camera for Surveys High Resolution Channel on the Hubble Space Telescope before, during and following the encounter of the Deep Impact spacecraft with comet 9P/Tempel 1. In addition, we serendipitously observed a natural outburst of the comet on 2005 June 14, whose characteristics may be compared with those of the ejecta produced by the impact. From the data we have obtained a measurement of the photometric size of the nucleus, determined the ejecta expansion velocity distribution, and followed the temporal evolution of the morphology of the visible brightness of the ejecta cloud. The high spatial resolution and image quality of the HRC images should make possible detailed kinematical modeling of the ejecta plume that may serve to constrain the physical properties of the ejecta particles.

\section{ACKNOWLEDGMENTS}

This work is based on observations with the NASA/ESA Hubble Space Telescope obtained at the Space Telescope Science Institute, which is operated by the Association of Universities for Research in Astronomy (AURA), Inc., under NASA contract NAS 5-26555. We thank Ian Jordan, Ron Gilliland, and Charles Proffitt (STScI) for the planning and successful execution of the HST program; Eddie Bergeron, Max Mutchler, Zolt Levay (STScI) and Ken Anderson (JHU) for the rapid response and production of properly corrected images; Cheryl Gundy, Lisa Frattare, Ray Villard, Mario Livio, and countless others at STScI for the July 4 logistics; and STScI/JPL for coordination of impact time before the end of 
$H S T$ visibility. This work was supported by grant GO-10144.01-A from the Space Telescope Science Institute.

\section{REFERENCES}

A'Hearn, M. F., Belton, M. J. S., Delamere, A., Blume, W. H. 2005a. Deep Impact: A Large-Scale Active Experiment on a Cometary Nucleus. Space Science Reviews 117, $1-21$.

A'Hearn, M. F., and 32 colleagues 2005b. Deep Impact: Excavating Comet Tempel 1. Science $310,258-264$.

Carcich, B., Elliott, G. 2006. View of Impact Site from Earth. Deep Impact Team Document.

Feldman, P. D., Lupu, R. E., McCandliss, S. R., Weaver, H. A., A'Hearn, M. F., Belton, M. J. S., Meech, K. J. 2006. Carbon Monoxide in Comet 9P/Tempel 1 before and after the Deep Impact Encounter. Astrophysical Journal (Letters) 647, L61.

Hanner, M. S., Giese, R. H., Weiss, K., Zerull, R. 1981. On the definition of albedo and application to irregular particles. Astronomy and Astrophysics 104, 42-46.

Harker, D. E., Woodward, C. E., Wooden, D. H. 2005. The Dust Grains from 9P/Tempel 1 Before and After the Encounter with Deep Impact. Science 310, 278-280.

Keller, H. U., and 11 colleagues 2005. Deep Impact Observations by OSIRIS Onboard the Rosetta Spacecraft. Science 310, 281-283.

Küppers, M., and 40 colleagues 2005. A large dust/ice ratio in the nucleus of comet 9P/Tempel 1. Nature 437, 987-990.

Lamy, P. L., Toth, I., A'Hearn, M. F., Weaver, H. A., Weissman, P. R. 2001. Hubble Space Telescope Observations of the Nucleus of Comet 9P/Tempel 1. Icarus 154, 337-344.

Lamy, P. L., Toth, I., Fernandez, Y. R., Weaver, H. A. 2004. The sizes, shapes, albedos, and colors of cometary nuclei, in Comets II, ed. M. C. Festou, H. A. Weaver, \& H. U. Keller (Tucson: Univ. of Arizona), 223-264.

Lara, L. M., Boehnhardt, H., Gredel, R., Gutiérrez, P. J., Ortiz, J. L., Rodrigo, R., VidalNuñez, M. J. 2006. Pre-impact monitoring of Comet 9P/Tempel 1, the Deep Impact target. Astronomy and Astrophysics 445, 1151-1157. 
Meech, K. J., and 208 colleagues 2005. Deep Impact: Observations from a Worldwide EarthBased Campaign. Science 310, 265-269.

Schleicher, D. G., Barnes, K. L., Baugh, N. F. 2006. Photometry and Imaging Results for Comet 9P/Tempel 1 and Deep Impact: Gas Production Rates, Postimpact Light Curves, and Ejecta Plume Morphology. Astronomical Journal 131, 1130-1137.

Schultz, P. H., Ernst, C. M., Anderson, J. L. B. 2005. Expectations for Crater Size and Photometric Evolution from the Deep Impact Collision. Space Science Reviews 117, 207-239.

Sirianni, M., and 13 colleagues 2005. The Photometric Performance and Calibration of the Hubble Space Telescope Advanced Camera for Surveys. Publications of the Astronomical Society of the Pacific 117, 1049-1112.

Sugita, S., and 22 colleagues 2005. Subaru Telescope Observations of Deep Impact. Science $310,274-278$. 
Table 1: Summary of ACS/HRC observations of comet 9P/Tempel 1. The HST visit number is the two digits preceding $* * *$ in the rootname. The exposure sequence for each visit is described in the text. Individual exposure IDs are given in the text where used. The parameters given correspond to the start time of the first exposure of each visit.

\begin{tabular}{|c|c|c|c|c|c|c|}
\hline $\begin{array}{c}\text { Visit } \\
\text { Rootname }\end{array}$ & Mode & Date & $\begin{array}{c}\text { Start } \\
\text { Time (UT) }\end{array}$ & $\begin{array}{c}r \\
(\mathrm{AU})\end{array}$ & $\begin{array}{c}\Delta \\
(\mathrm{AU})\end{array}$ & $\begin{array}{c}\text { Phase } \\
\text { Angle }\left(^{\circ}\right)\end{array}$ \\
\hline \multicolumn{7}{|c|}{ June pre-impact } \\
\hline $\mathrm{J} 8 \mathrm{Z} 301 * * *$ & HRC F606W + PR200L & 2005-06-13 & $05: 36: 11$ & 1.522 & 0.799 & 37.7 \\
\hline $\mathrm{J} 8 \mathrm{Z} 302^{* * *}$ & HRC F606W + HRC-512 & 2005-06-13 & $15: 11: 58$ & 1.522 & 0.800 & 37.8 \\
\hline $\mathrm{J} 8 \mathrm{Z} 303^{* * *}$ & HRC F606W + PR200L & 2005-06-14 & $07: 11: 36$ & 1.521 & 0.803 & 37.9 \\
\hline $\mathrm{J} 8 \mathrm{Z} 304^{* * *}$ & HRC F606W + HRC-512 & 2005-06-14 & 14:09:20 & 1.521 & 0.804 & 38.0 \\
\hline \multicolumn{7}{|c|}{ Impact epoch } \\
\hline J9A801*** & HRC F606W + PR200L & 2005-07-02 & $00: 32: 22$ & 1.507 & 0.883 & 40.7 \\
\hline J9A $803^{* * *}$ & HRC F606W + PR200L & 2005-07-02 & $22: 55: 32$ & 1.506 & 0.887 & 40.8 \\
\hline J9A805*** & HRC-512 F606W (impact) & 2005-07-04 & $05: 19: 10$ & 1.506 & 0.894 & 40.9 \\
\hline J9A806*** & HRC F606W + PR200L & 2005-07-04 & $06: 54: 26$ & 1.506 & 0.894 & 40.9 \\
\hline J9A $808^{* * *}$ & HRC F606W + PR200L & 2005-07-04 & 10:06:20 & 1.506 & 0.895 & 41.0 \\
\hline J9A810*** & HRC F606W + PR200L & $2005-07-05$ & $00: 29: 54$ & 1.506 & 0.898 & 41.0 \\
\hline $\mathrm{J} 9 \mathrm{~A} 812^{* * *}$ & HRC F606W + PR200L & 2005-07-11 & 18:03:11 & 1.508 & 0.935 & 41.4 \\
\hline J9A $822^{* * *}$ & HRC F606W + PR200L & $2005-07-16$ & $22: 55: 25$ & 1.511 & 0.966 & 41.6 \\
\hline
\end{tabular}




\section{FIGURE CAPTIONS}

Fig. 1.- Left: Mean of four exposures from visit 01, J9A801DZQ, J9A801E0Q, J9A801E5Q, and J9A801E6Q, each 140 s, taken about 54 hours pre-impact. Right, Mean of exposures J9A806EQQ and J9A806ERQ, both $140 \mathrm{~s}$, taken approximately one hour after impact. The legend in the right panel gives the start time of the first exposure.

Fig. 2.- Azimuthally averaged radial profile of an image taken immediately prior to impact (J9A805EAQ). The green line is a dust coma model (proportional to $\rho^{-1}$ ) convolved with the instrumental point-spread function while the red line is the difference between the data and the coma model, which yields an estimate for the contribution from the nucleus. The pixel size is $0 . " 025$.

Fig. 3.- Images of the natural outburst on 2005 June 14 created by taking the ratio of exposures from the fourth visit to a mean of two exposures obtained $\sim 7$ hours earlier (J8Z303G2Q and J8Z303G3Q). The two images were taken 67 minutes apart. Left: Mean of J8Z304I7Q and J8Z304I8Q. Right: Mean of J8Z304IBQ and J8Z304ICQ. All exposures were $300 \mathrm{~s}$. The exposure start times are indicated in the figure. The "tongue depresser" feature in the NW quadrant is due to an occulting finger in the HRC.

Fig. 4.- Contour plots of the two ratio images shown in Fig. 3. The fan appears symmetric about a position angle of $311^{\circ}$, while the position angle of the Sun is $295^{\circ}$. Semi-circles, centered on the nucleus are shown, of radius $2330 \mathrm{~km}$ and $2910 \mathrm{~km}$, respectively, for the left and right plots.

Fig. 5.- Ratio images of the 40-s exposures taken during and in the $13.6 \mathrm{~min}$ immediately following impact to the mean of the last four prior exposures. The first exposure, J9A805EBQ began $23 \mathrm{~s}$ before the time of impact as seen from Earth. The exposure mid-points are $75 \mathrm{~s}$ apart.

Fig. 6.- Contour plots of four of the ratio images shown in Fig. 5.

Fig. 7.- Images created by taking the ratio of post-impact exposures to the pre-impact image shown in the left panel of Fig. 1. Left: Mean of J9A806EQQ and J9A806ERQ, each 140 s. Right: Mean of J9A806EWQ and J9A806EXQ, each 140 s. The exposure start times are indicated in the figure. The exposure mid-points are 68.2 (left) and $92.2 \mathrm{~min}$ (right) after impact. 
Fig. 8. - Same as Fig. 7. Left: Mean of J9A808F9Q and J9A808FAQ, each 140 s. Right: Mean of J9A808FFQ and J9A808FGQ, each 140 s. The exposure mid-points are 260.1 (left) and 284.1 min (right) after impact.

Fig. 9.- Contour plots of the two ratio images shown in Fig. 7. The fan appears symmetric about a position angle of $240^{\circ}$. Semi-circles, centered on the nucleus are shown, of radius $1210 \mathrm{~km}$ and $1610 \mathrm{~km}$, respectively, for the left and right plots.

Fig. 10.- Radial profiles of the pre- and post-impact images used in the ratio contour plots shown in Fig. 9. The range of position angle is 180 to $300^{\circ}$. Black: pre-impact; Red: postimpact; Blue: difference between post- and pre-impact, multiplied by radial distance (in pixel units). The dashed curve gives the fraction of total flux (multiplied by 1000) remaining outside a given radial distance.

Fig. 11.- Normalized velocity distributions derived from the blue curves in Fig. 10 (Black: left panel; Red: Right panel).

Fig. 12.- Azimuthal profiles of the pre-impact $(\mathrm{red}, \times 10)$ and post-impact (black) images used in the ratio contour plot shown in the left panel of Fig. 9. The range of radial distance is 200 to $650 \mathrm{~km}$.

Fig. 13. - Light curves based on aperture photometry. Left: impact orbit. Right: the following $H S T$ orbit. The pre-impact count rates are $1.76,2.78,3.95$, and 6.96 , all $\times 10^{3}$ counts s $^{-1}$, for the 40, 80, 160, and $400 \mathrm{~km}$ radius apertures, respectively, and have been subtracted from the data points.

Fig. 14.- Light curves based on aperture photometry through $20 \mathrm{~h}$ following impact. The pre-impact values have not been subtracted as in Fig. 13 . 


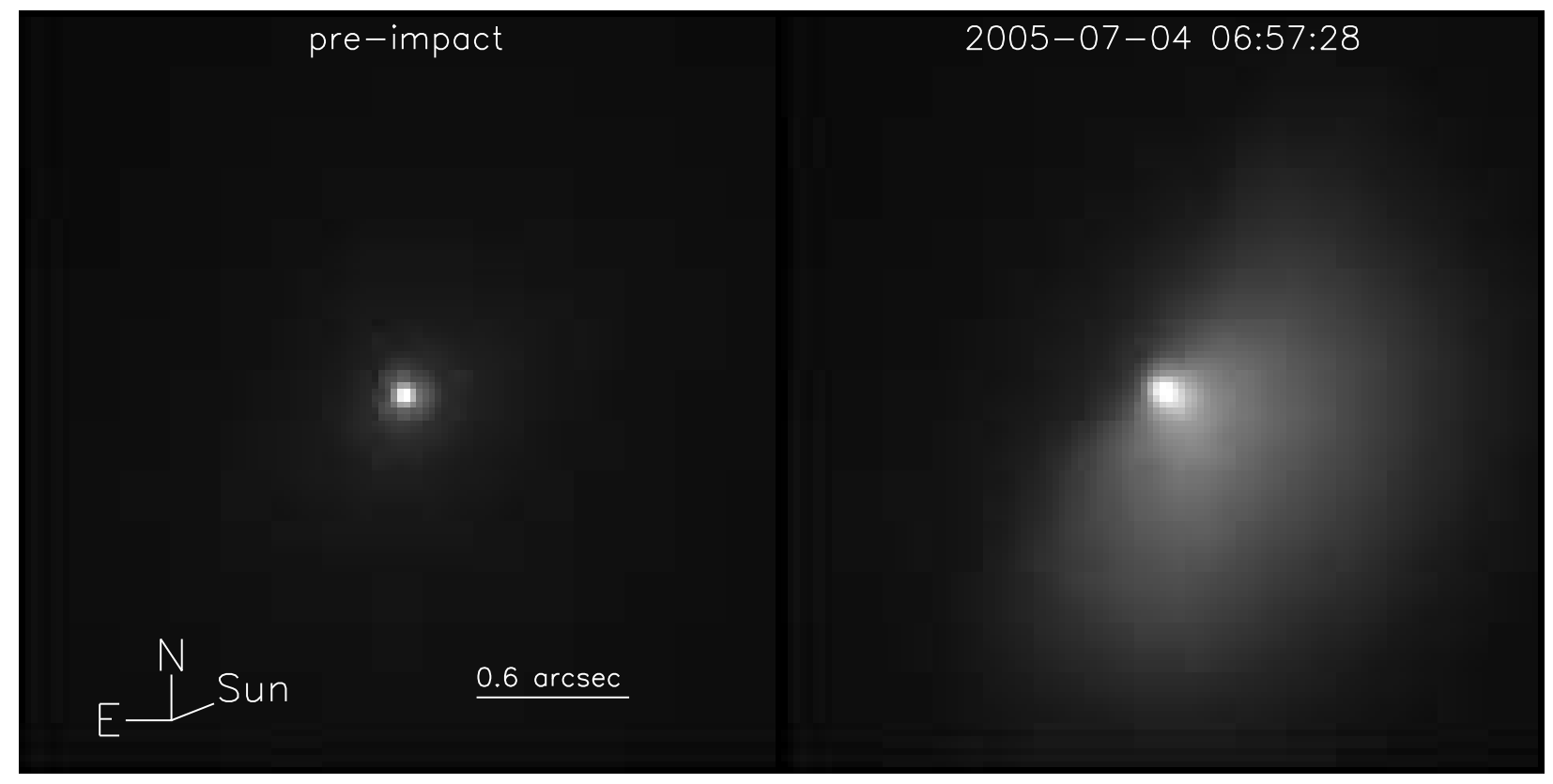

Fig. 1.- Left: Mean of four exposures from visit 01, J9A801DZQ, J9A801E0Q, J9A801E5Q, and J9A801E6Q, each $140 \mathrm{~s}$, taken about 54 hours pre-impact. Right, Mean of exposures J9A806EQQ and J9A806ERQ, both $140 \mathrm{~s}$, taken approximately one hour after impact. The legend in the right panel gives the start time of the first exposure. 


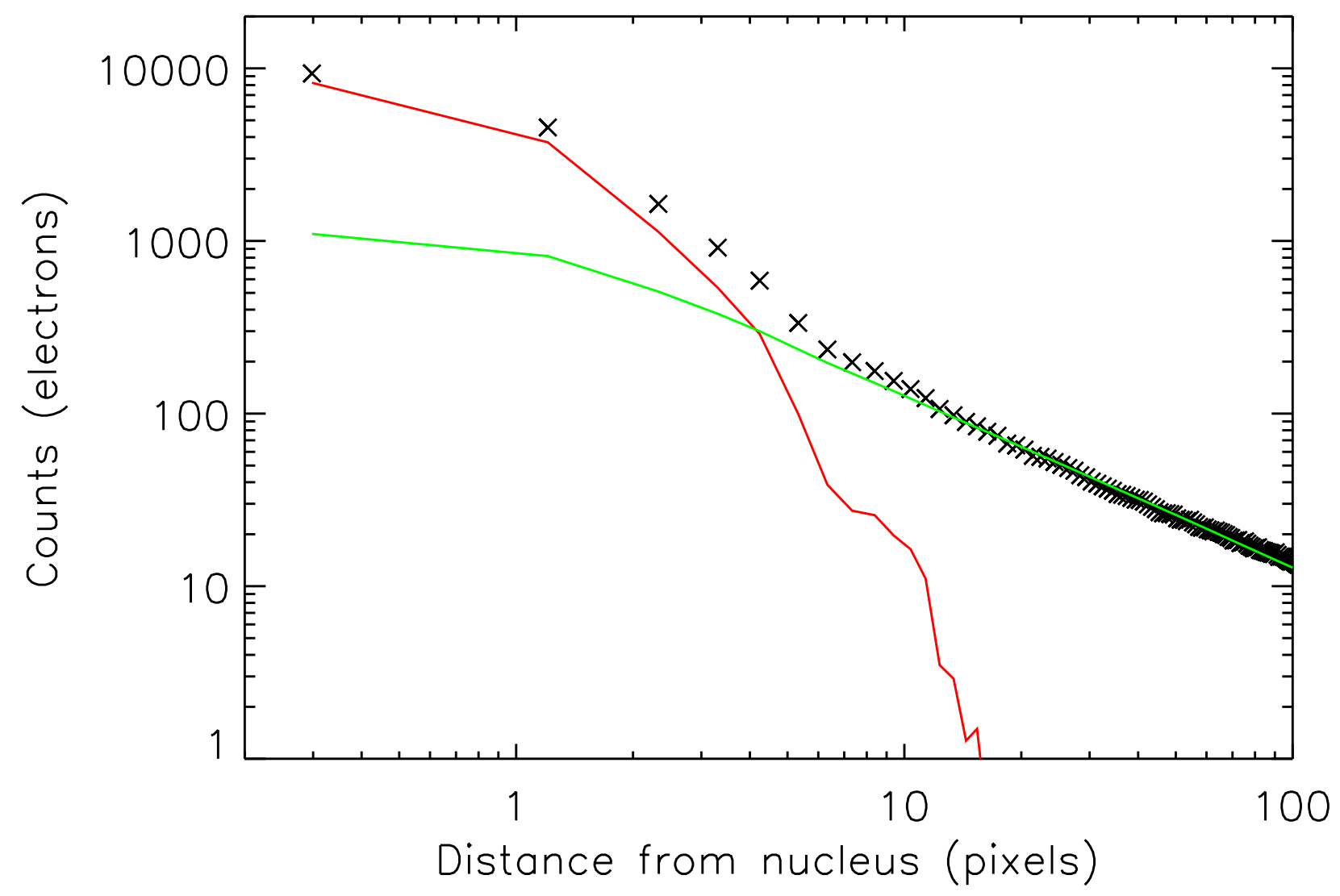

Fig. 2.- Azimuthally averaged radial profile of an image taken immediately prior to impact (J9A805EAQ). The green line is a dust coma model (proportional to $\rho^{-1}$ ) convolved with the instrumental point-spread function while the red line is the difference between the data and the coma model, which yields an estimate for the contribution from the nucleus. The pixel size is $0 . " 025$. 


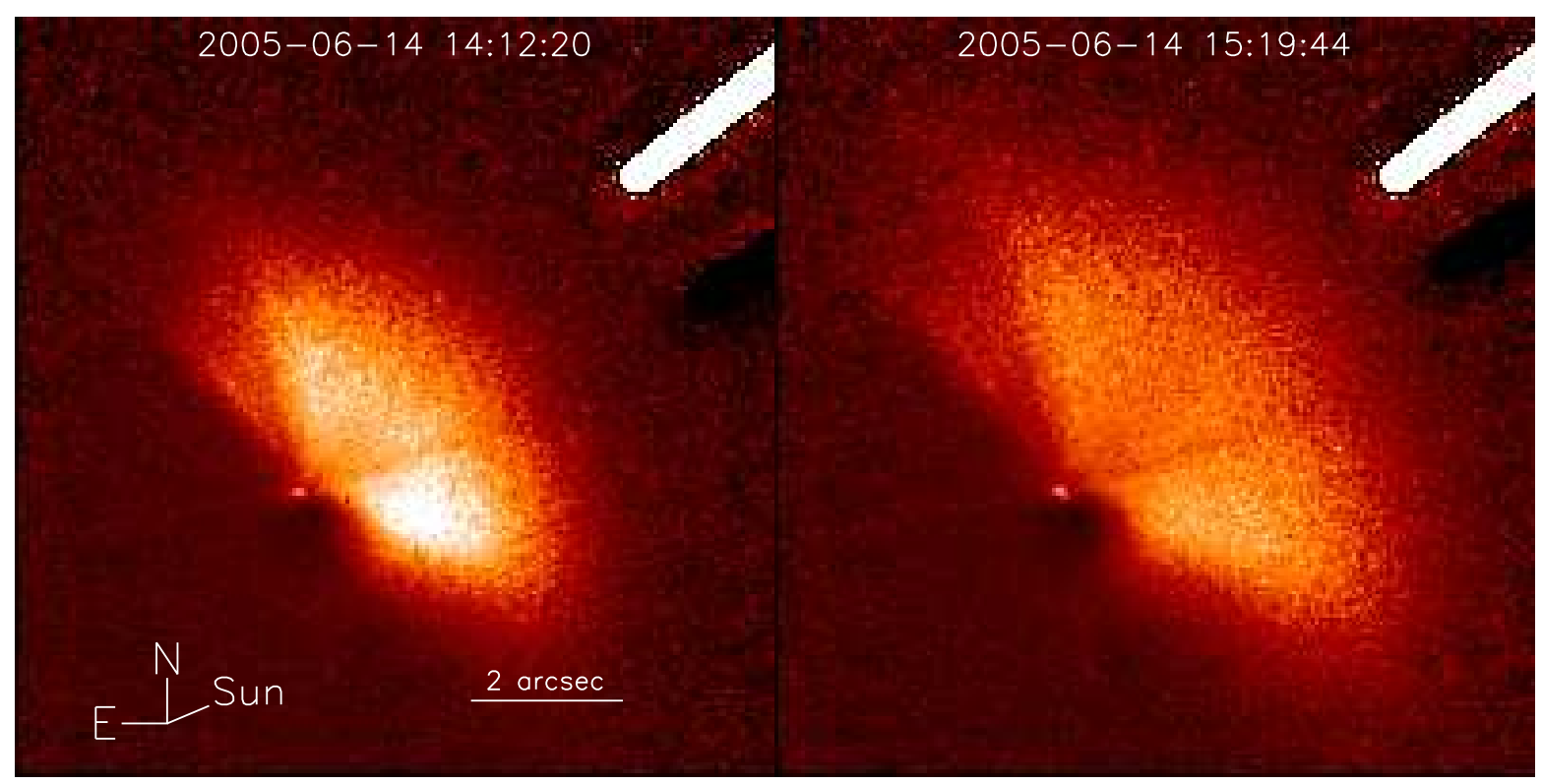

Fig. 3.- Images of the natural outburst on 2005 June 14 created by taking the ratio of exposures from the fourth visit to a mean of two exposures obtained $\sim 7$ hours earlier (J8Z303G2Q and J8Z303G3Q). The two images were taken 67 minutes apart. Left: Mean of J8Z304I7Q and J8Z304I8Q. Right: Mean of J8Z304IBQ and J8Z304ICQ. All exposures were $300 \mathrm{~s}$. The exposure start times are indicated in the figure. The "tongue depresser" feature in the NW quadrant is due to an occulting finger in the HRC. 

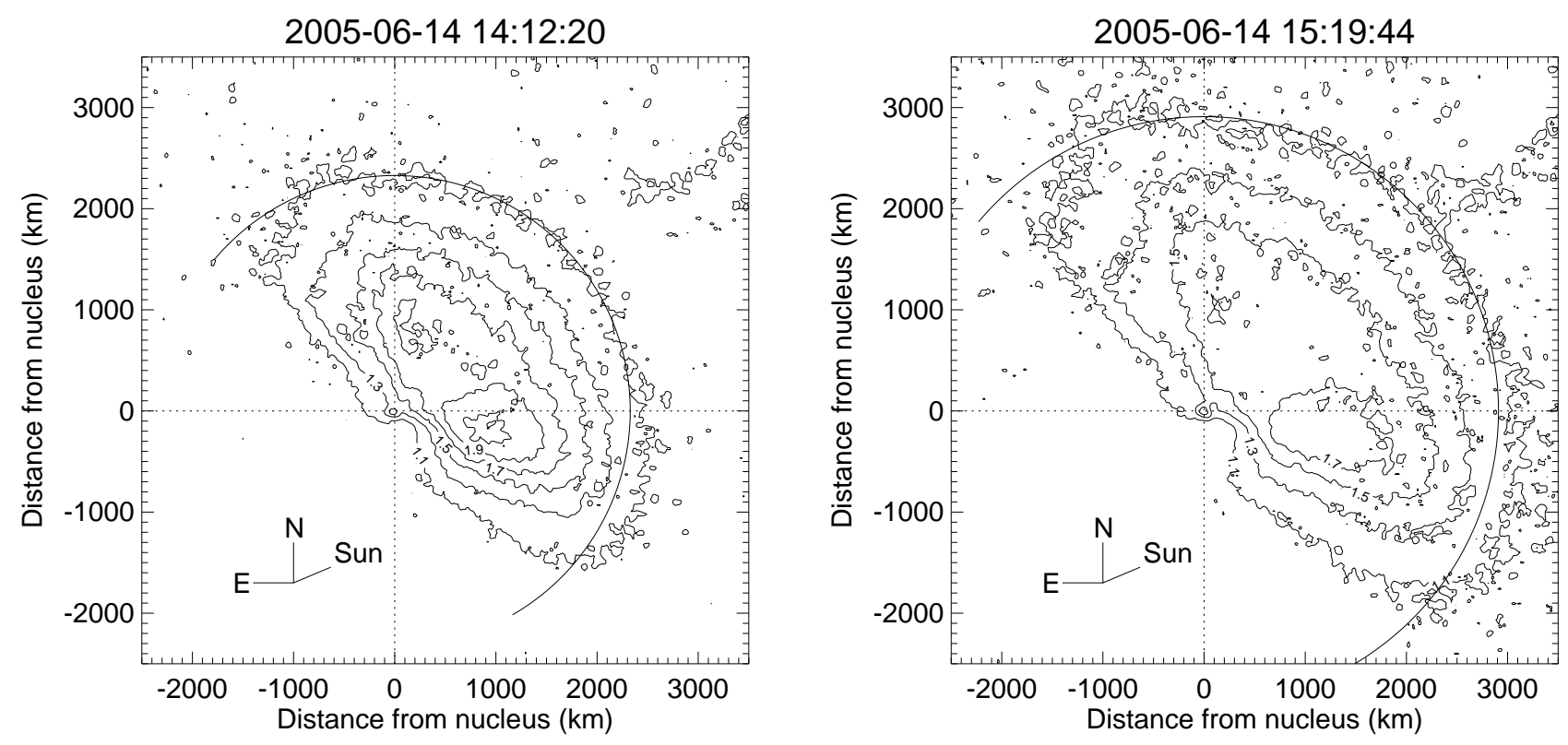

Fig. 4.- Contour plots of the two ratio images shown in Fig. 3. The fan appears symmetric about a position angle of $311^{\circ}$, while the position angle of the Sun is $295^{\circ}$. Semi-circles, centered on the nucleus are shown, of radius $2330 \mathrm{~km}$ and $2910 \mathrm{~km}$, respectively, for the left and right plots. 


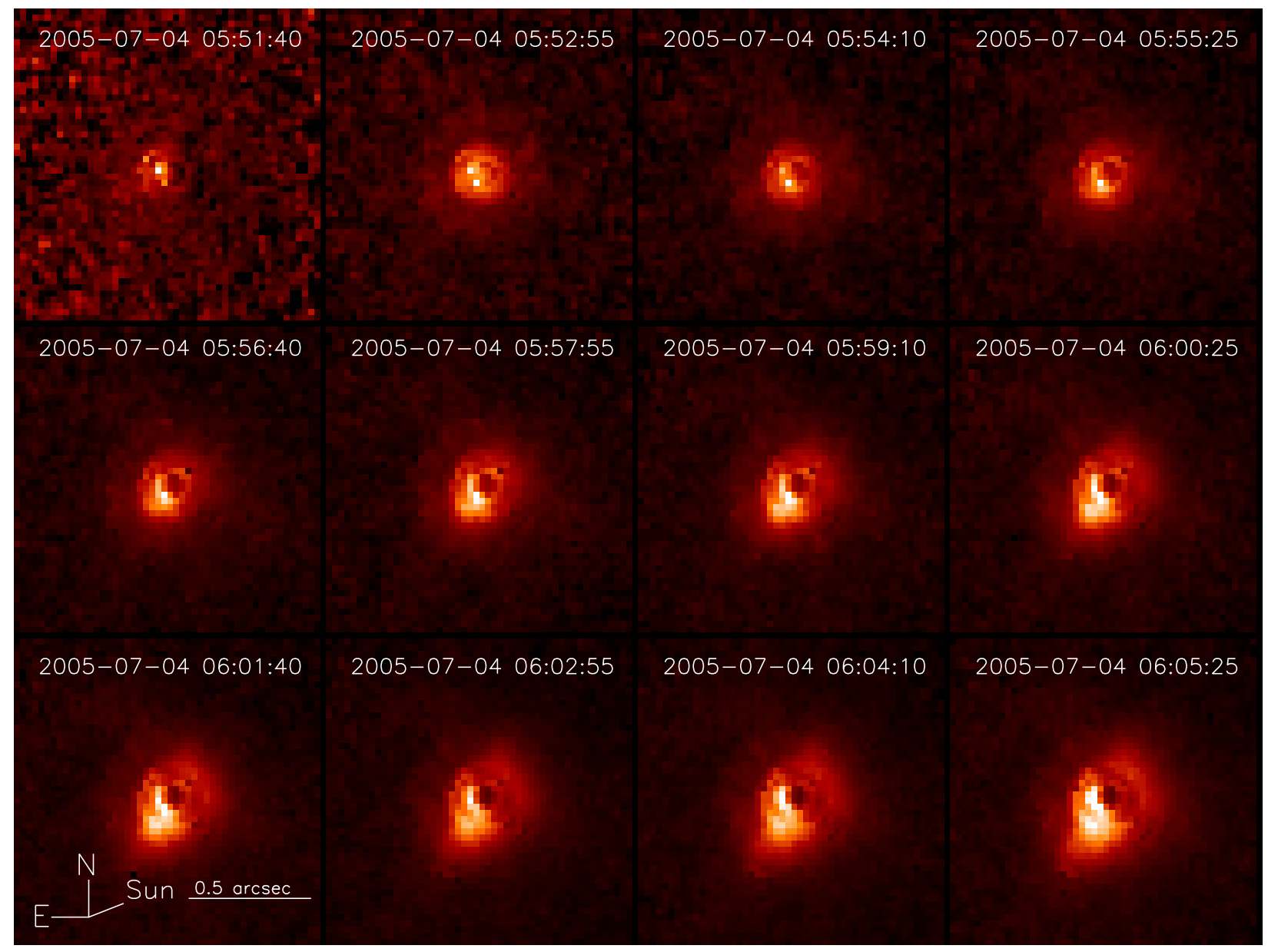

Fig. 5.- Ratio images of the 40-s exposures taken during and in the 13.6 min immediately following impact to the mean of the last four prior exposures. The first exposure, J9A805EBQ began $23 \mathrm{~s}$ before the time of impact as seen from Earth. The exposure mid-points are $75 \mathrm{~s}$ apart. 

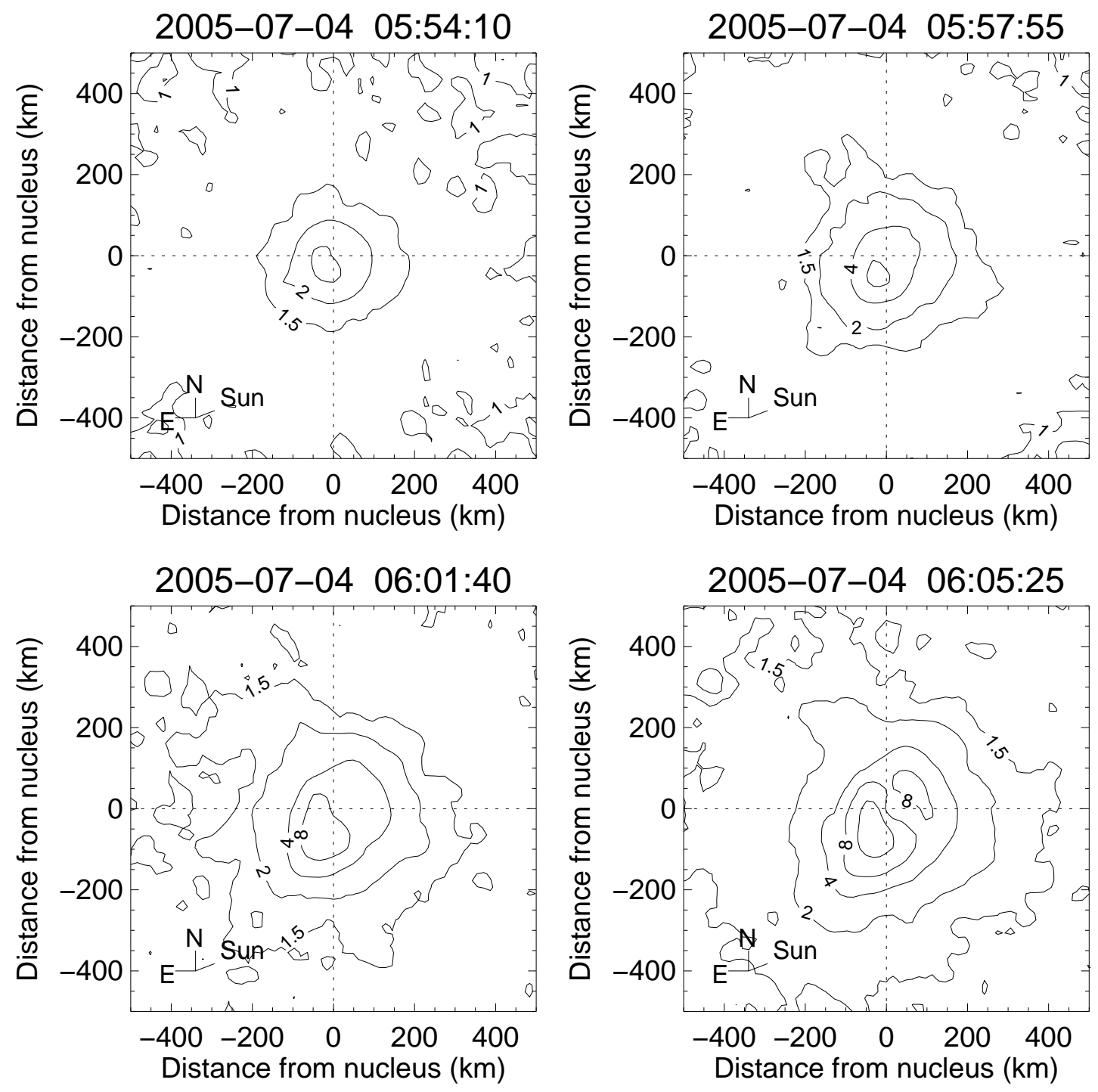

Fig. 6.- Contour plots of four of the ratio images shown in Fig. 5. 
Fig. 7.- Images created by taking the ratio of post-impact exposures to the pre-impact image shown in the left panel of Fig. 1. Left: Mean of J9A806EQQ and J9A806ERQ, each 140 s. Right: Mean of J9A806EWQ and J9A806EXQ, each 140 s. The exposure start times are indicated in the figure. The exposure mid-points are 68.2 (left) and $92.2 \mathrm{~min}$ (right) after impact. 


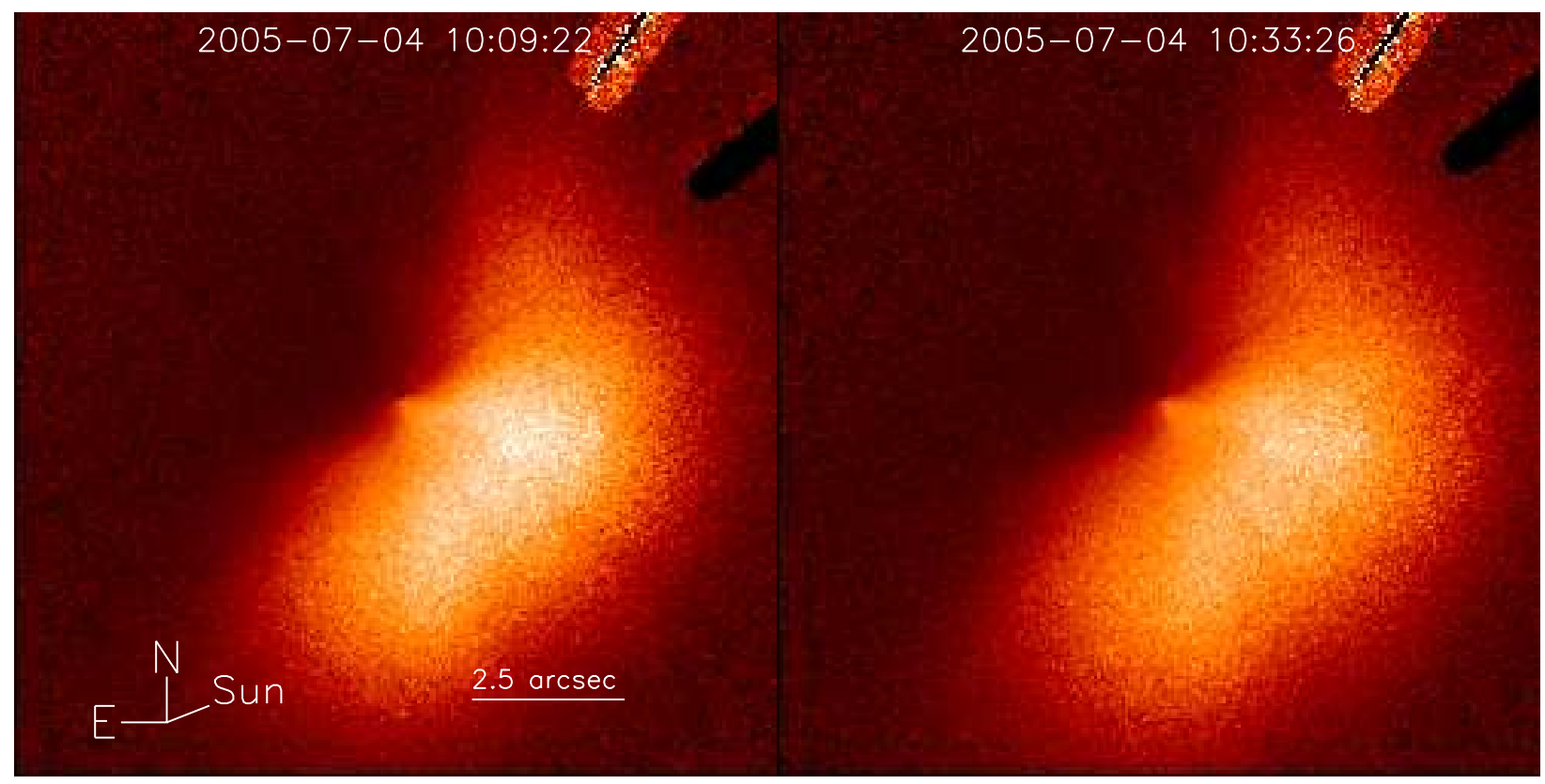

Fig. 8.- Same as Fig. 7. Left: Mean of J9A808F9Q and J9A808FAQ, each 140 s. Right: Mean of J9A808FFQ and J9A808FGQ, each 140 s. The exposure mid-points are 260.1 (left) and $284.1 \mathrm{~min}$ (right) after impact. 

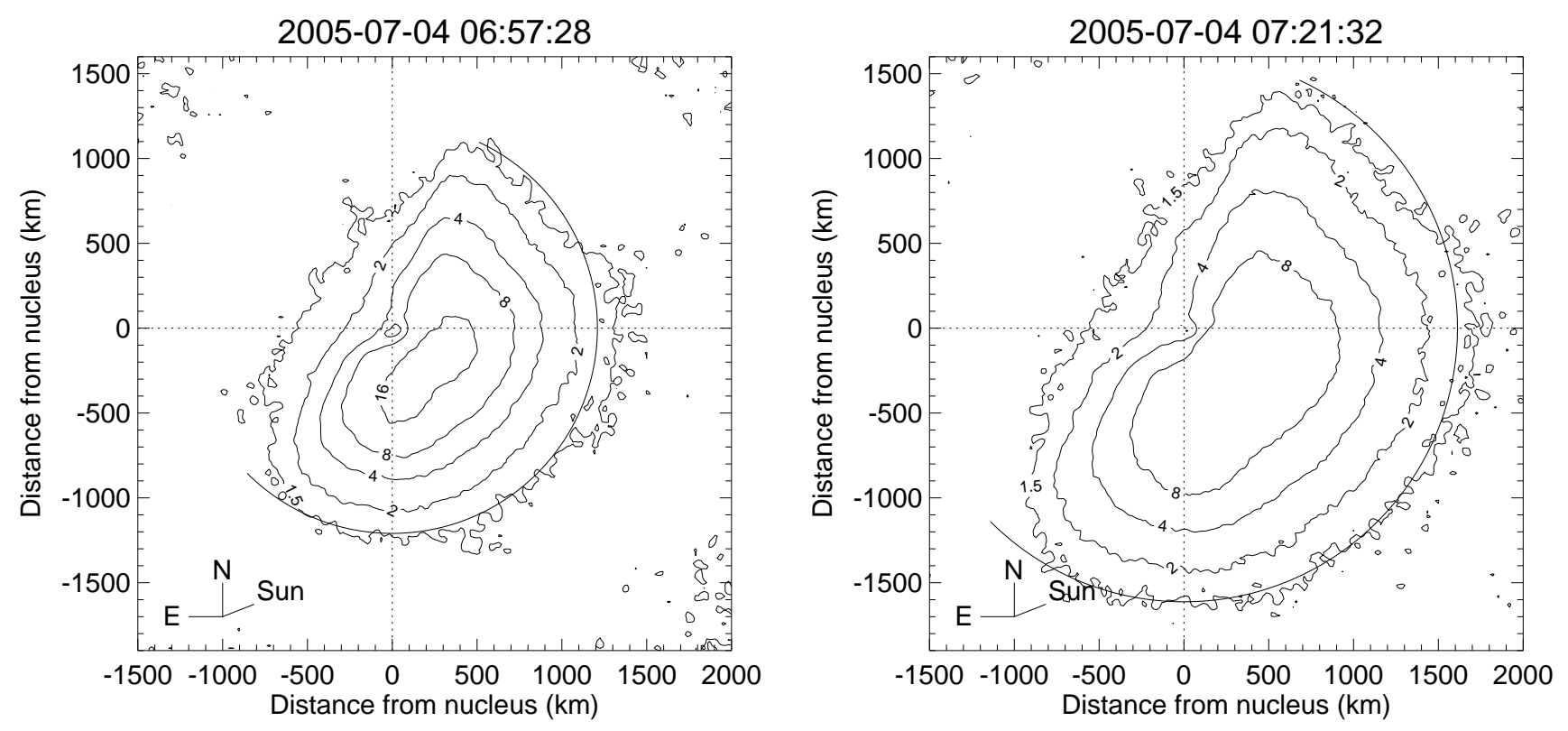

Fig. 9.- Contour plots of the two ratio images shown in Fig. 7. The fan appears symmetric about a position angle of $240^{\circ}$. Semi-circles, centered on the nucleus are shown, of radius $1210 \mathrm{~km}$ and $1610 \mathrm{~km}$, respectively, for the left and right plots. 

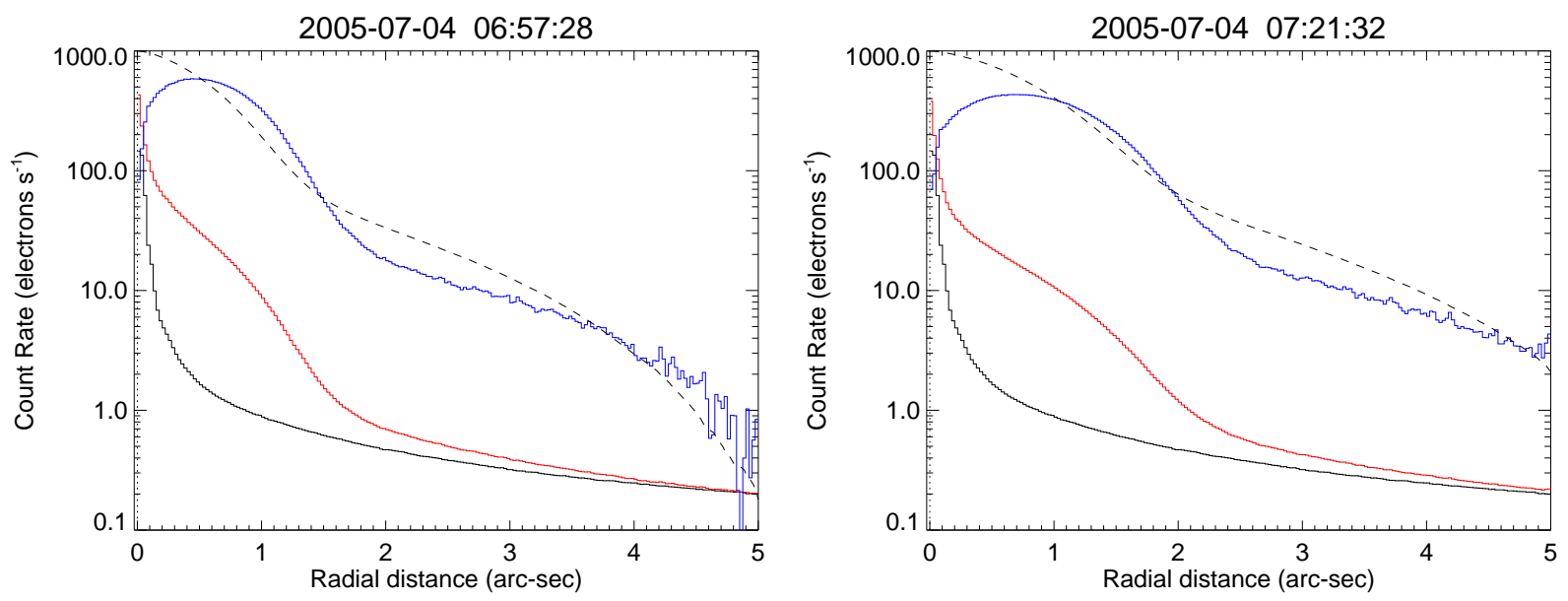

Fig. 10.- Radial profiles of the pre- and post-impact images used in the ratio contour plots shown in Fig. 9. The range of position angle is 180 to $300^{\circ}$. Black: pre-impact; Red: postimpact; Blue: difference between post- and pre-impact, multiplied by radial distance (in pixel units). The dashed curve gives the fraction of total flux (multiplied by 1000) remaining outside a given radial distance. 


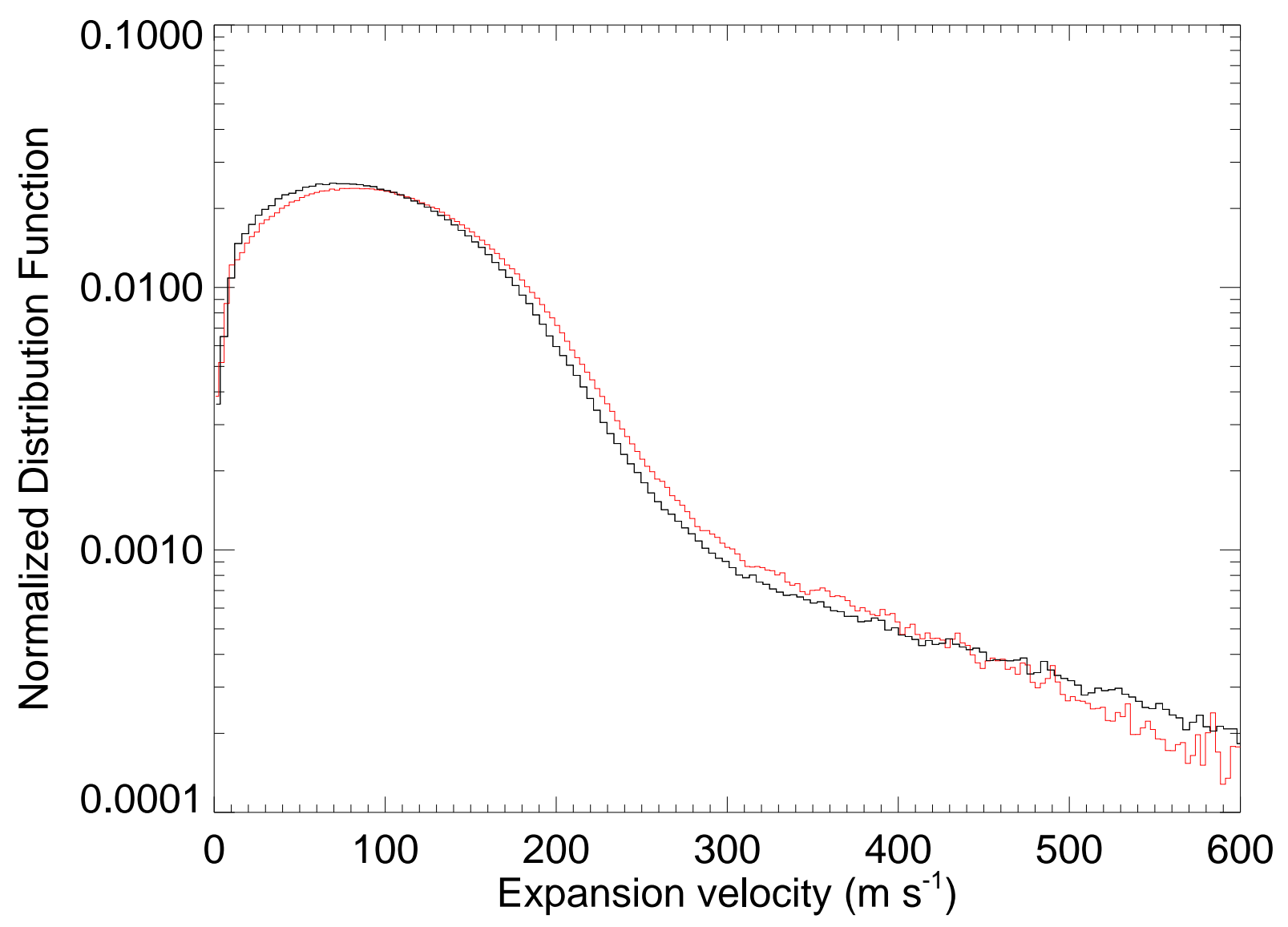

Fig. 11.- Normalized velocity distributions derived from the blue curves in Fig. 10 (Black: left panel; Red: Right panel). 


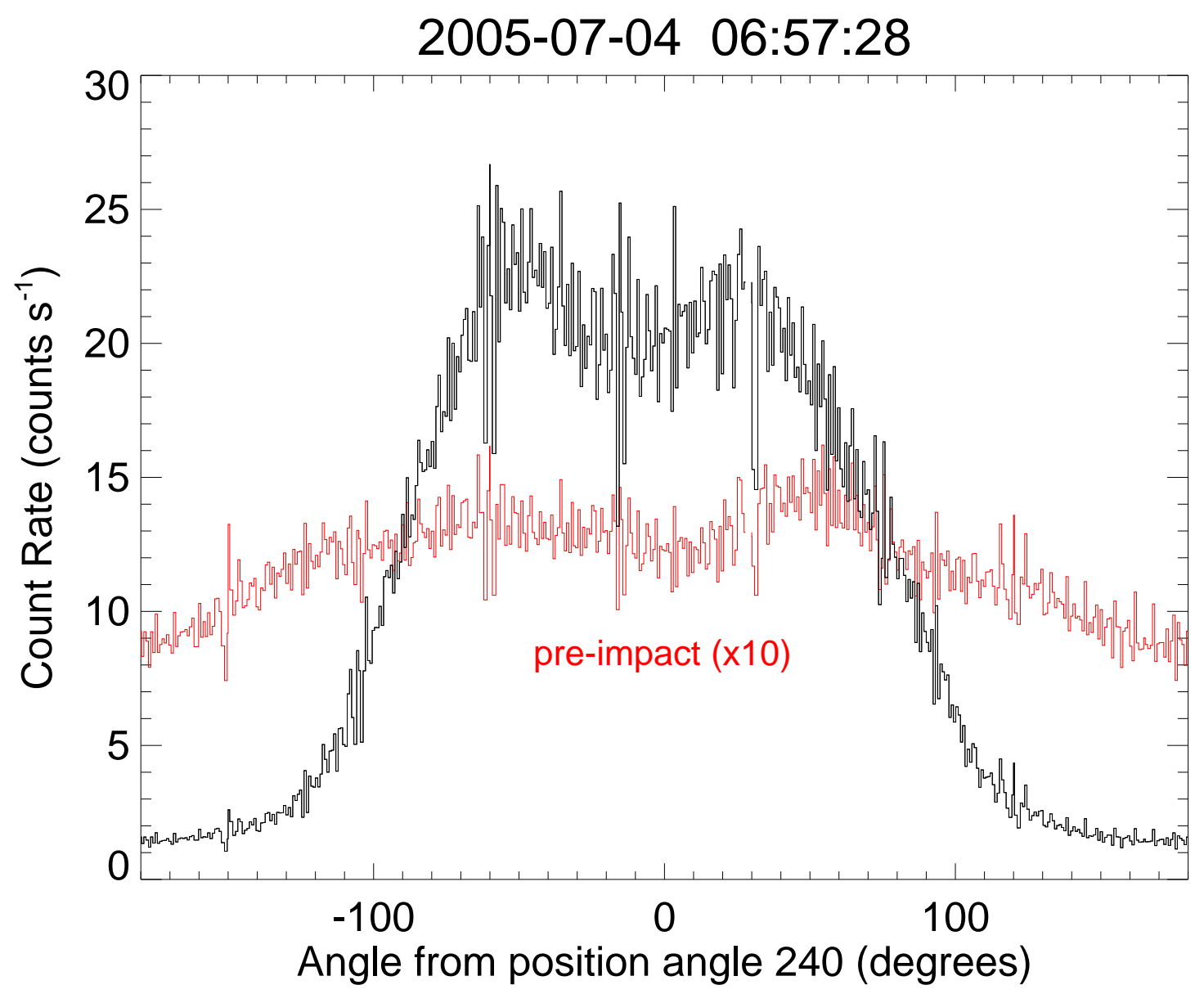

Fig. 12.- Azimuthal profiles of the pre-impact (red, $\times 10$ ) and post-impact (black) images used in the ratio contour plot shown in the left panel of Fig. 9. The range of radial distance is 200 to $650 \mathrm{~km}$. 

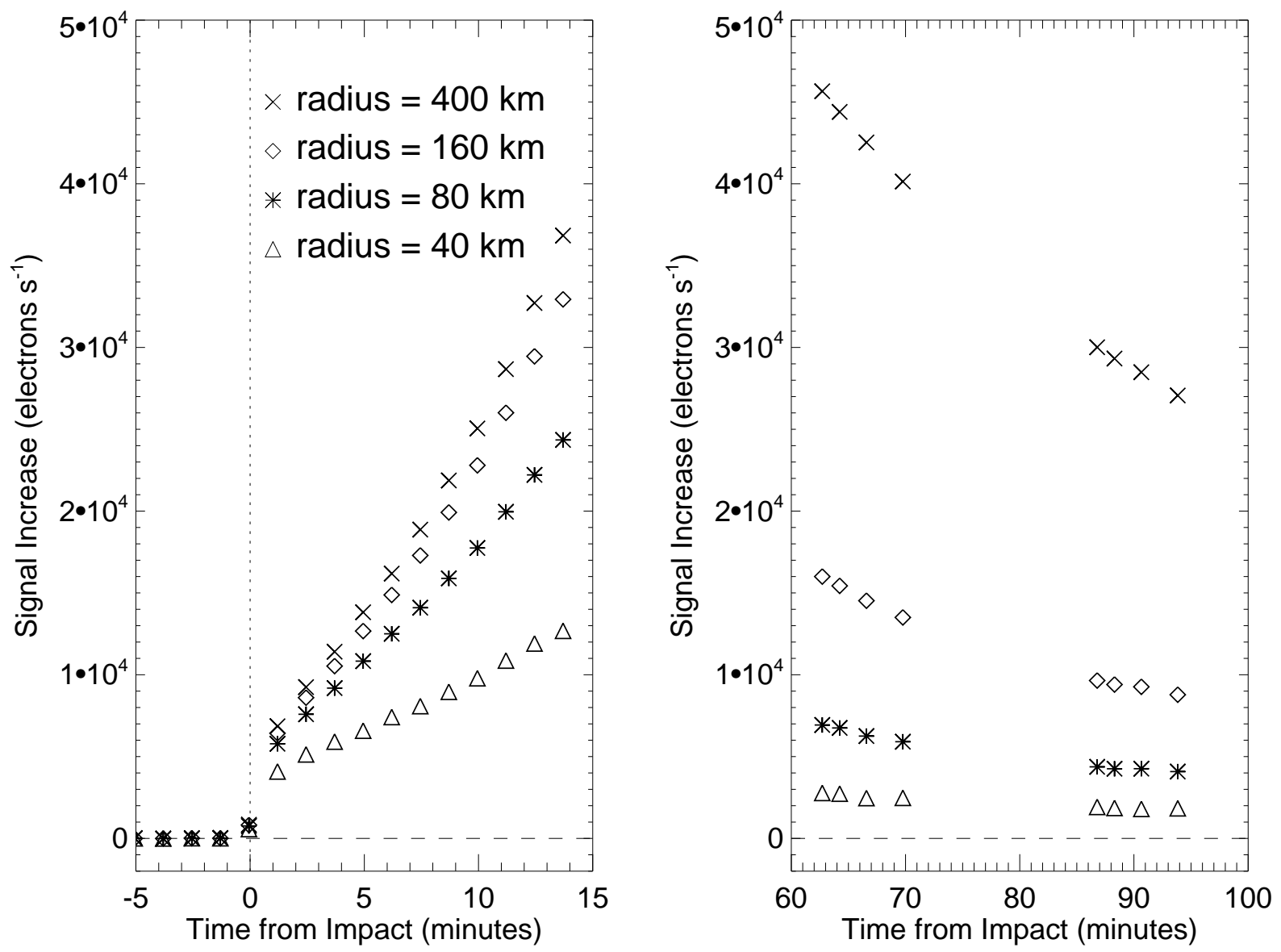

Fig. 13. - Light curves based on aperture photometry. Left: impact orbit. Right: the following HST orbit. The pre-impact count rates are $1.76,2.78,3.95$, and 6.96 , all $\times 10^{3}$ counts s$^{-1}$, for the 40, 80, 160, and $400 \mathrm{~km}$ radius apertures, respectively, and have been subtracted from the data points. 


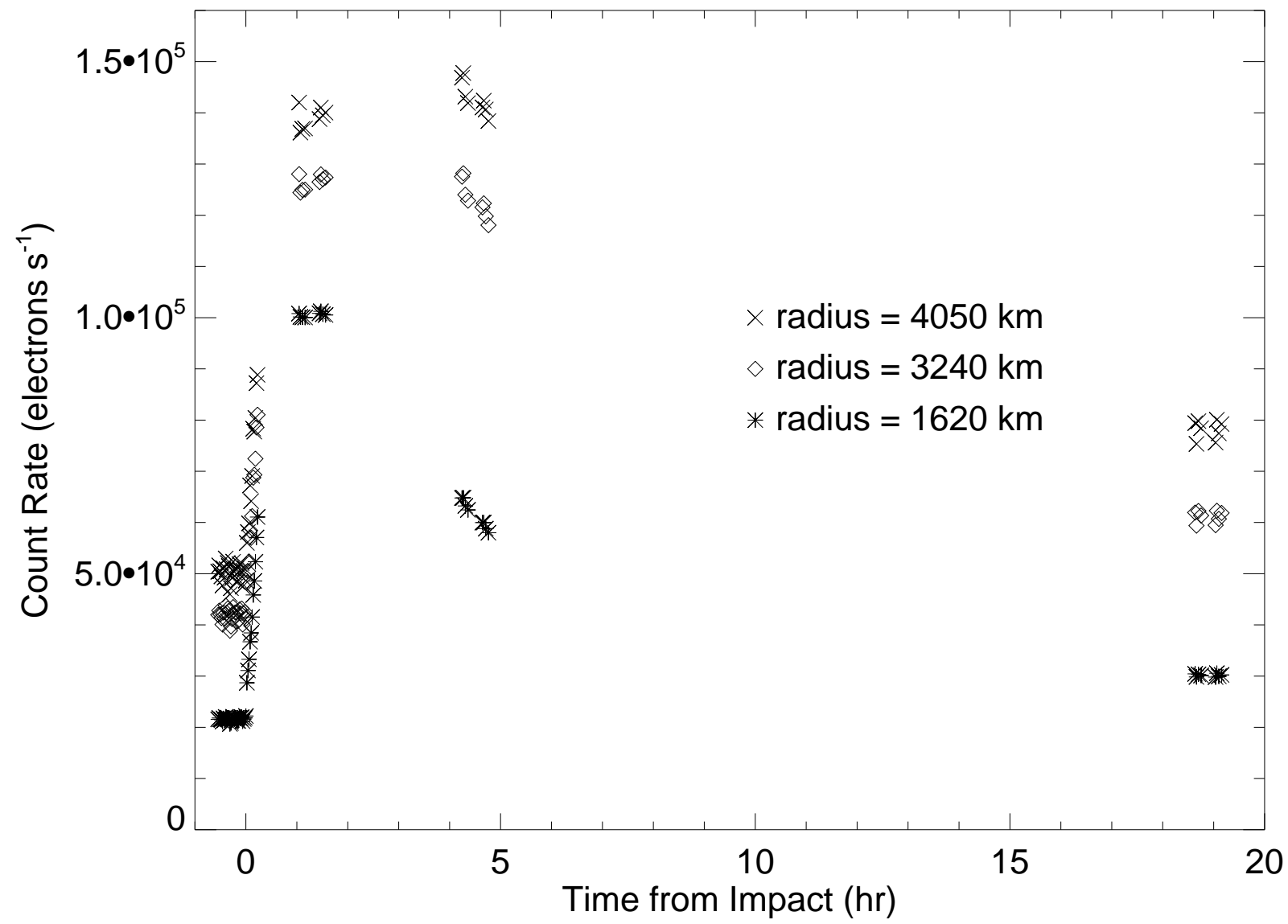

Fig. 14.- Light curves based on aperture photometry through $20 \mathrm{~h}$ following impact. The pre-impact values have not been subtracted as in Fig. 13. 